IOS Press

\title{
Review
}

\section{Management of Adrenal Insufficiency Risk After Long-term Systemic Glucocorticoid Therapy in Duchenne Muscular Dystrophy: Clinical Practice Recommendations}

\author{
Sasigarn A. Bowden ${ }^{\mathrm{a}, *}$, Anne M. Connolly ${ }^{\mathrm{b}}$, Kathi Kinnett ${ }^{\mathrm{c}}$ and Philip S. Zeitler ${ }^{\mathrm{d}}$ \\ ${ }^{a}$ Division of Endocrinology, Department of Pediatrics, Nationwide Children's Hospital/The Ohio State \\ University College of Medicine, Columbus, Ohio, USA \\ ${ }^{\mathrm{b}}$ Department of Neurology, Washington University School of Medicine in Saint Louis, St. Louis, Missouri, USA \\ ${ }^{\mathrm{c}}$ Parent Project Muscular Dystrophy, Hackensack, New Jersey, USA \\ ${ }^{\mathrm{d}}$ Department of Pediatrics, Division of Endocrinology, University of Colorado School of Medicine, \\ Aurora, Colorado, USA
}

\begin{abstract}
Long-term glucocorticoid therapy has improved outcomes in patients with Duchenne muscular dystrophy. However, the recommended glucocorticoid dosage suppresses the hypothalamic-pituitary-adrenal axis, leading to adrenal insufficiency that may develop during severe illness, trauma or surgery, and after discontinuation of glucocorticoid therapy. The purpose of this review is to highlight the risk of adrenal insufficiency in this patient population, and provide practical recommendations for management of adrenal insufficiency, glucocorticoid withdrawal, and adrenal function testing. Strategies to increase awareness among patients, families, and health care providers are also discussed.
\end{abstract}

Keywords: Muscular dystrophy, adrenal crisis, adrenal suppression, cortisol, prednisone, deflazacort, ACTH

\section{INTRODUCTION}

Duchenne muscular dystrophy (DMD) is an $\mathrm{X}$-linked recessive neuromuscular disease, characterized by progressive muscle weakness, with eventual loss of ambulation and premature death [1]. Incidence of DMD has been reported as one case per 3500-5000

\footnotetext{
*Correspondence to: Sasigarn A. Bowden, MD, Nationwide Children's Hospital, Division of Endocrinology, 700 Children's Drive, Columbus, OH 43205, USA. Tel.: +1 614722 4118; Fax: +1614722 4440; E-mail: sasigarn.bowden@ nationwidechildrens. org.
}

male newborns [2-5]. Therapy with glucocorticoids (GC) improves muscle strength, prolongs ambulation and maintains cardiac muscle and pulmonary function [6, 7]. Nevertheless, prolonged use of daily GC therapy is associated with significant side effects including cushingoid features, obesity, abnormal glucose tolerance, growth retardation, delayed puberty, hypertension, adverse behavioral changes and increased fracture risk [8-10]. Often overlooked, however, is the iatrogenic suppression of the hypothalamic-pituitary-adrenal (HPA) axis, i.e. adrenal suppression, with resultant secondary adrenal insufficiency (AI). 
Secondary AI is different from primary AI or Addison disease in that the primary AI is caused by destruction or dysfunction of the adrenal gland (primary adrenal gland disorder), whereas, secondary AI is due to deficient pituitary adrenocorticotrophic hormone (ACTH) secretion, or suppressed ACTH secretion secondary to chronic GC therapy. Acute AI or adrenal crisis is a life-threatening condition that may develop during physiological stress events when patients decompensate and are not able to meet the increased demand of cortisol. AI secondary to chronic GC therapy may persist long after exogenous GC therapy is discontinued. AI in boys and men with DMD may occur in several scenarios, including abrupt cessation of GC, during gastrointestinal infection or febrile illness with emesis that inhibits normal absorption of GC, or when patients undergo surgery and do not receive GC stress dose coverage peri-operatively. The prevalence of symptomatic AI or adrenal crisis in patients with DMD is unknown as routine screening is generally not performed and no studies have systematically addressed this issue. Evidence suggests that morbidity and mortality associated with acute AI or adrenal crisis are still high among individuals with AI from various disorders $[11,12]$. Some unexplained deaths among patients with DMD during acute illnesses have been speculated to be related to adrenal crisis rather than cardiac complications or other acute stressor/illness. One young man with DMD died following an orthopedic surgery for humerus and femur fractures when he developed hypotension postoperatively without the benefit of GC supplementation [13]. This patient had been on chronic daily GC for many years, thus, this medically significant stress event occurred during a time that adrenal function was likely suppressed. It was determined only after a careful retrospective chart review that he likely suffered from adrenal crisis, because he had not received consistent GC replacement during his hospitalization [13]. This led to the Parent Project Muscular Dystrophy's development and publication of the "PJ Nicholoff Steroid Protocol" (www.ParentProjectMD.org/PJ). This young man's story serves as an important reminder of the real risk of AI in people living with DMD who are taking daily GC. This story also highlights the need to increase awareness of adrenal suppression among health care providers and the need for GC management during stress events in patients with DMD.

The purpose of this review is to provide physicians and other health care professionals caring for patients with DMD an overview of the physiology of the HPA axis, the pharmacology of $\mathrm{GC}$, management of AI and acute adrenal crisis, and practical recommendations for GC withdrawal and adrenal function testing. Strategies to increase awareness among patients, families, and health care providers are also discussed. Noting that research on AI in patients with DMD is lacking, it is our hope that this review will serve as a framework for future research and to facilitate AI surveillance/monitoring, with the goal of improving recognition and preventing complications. Additional work is needed to better understand the true epidemiology of AI in patients with DMD on long-term GC therapy.

\section{The physiology of the HPA Axis}

The HPA axis is a central stress response system that stimulates production and release of cortisol from the adrenal gland. Cortisol acts to support vascular tone, increases availability of glucose, and maintains intravascular volume. When the body encounters a stressor (e.g., surgery, trauma), the hypothalamus is activated and releases corticotropinreleasing hormone (CRH), which acts on the anterior pituitary gland to stimulate release of adrenocorticotropin hormone (ACTH). ACTH then activates the adrenal gland to release cortisol. The HPA axis has a negative feedback mechanism, such that increased circulating cortisol levels inhibit both the hypothalamus and pituitary glands to suppress release of CRH and ACTH (Fig. 1). Conversely, insufficient cortisol stimulates increased release of $\mathrm{CRH}$ and ACTH.

Normal basal secretion of cortisol from the adrenal gland in unstressed individuals is $5-7 \mathrm{mg} / \mathrm{m}^{2} /$ day for adolescents [14] or $8-10 \mathrm{mg} /$ day for adults [15]. This basal secretion is equivalent to approximately $10-12 \mathrm{mg} / \mathrm{m}^{2} /$ day of hydrocortisone administered orally to compensate for the incomplete bioavailability secondary to intestinal absorption and hepatic metabolism [11]. The basal secretion increases 3-5 times during minor illnesses or surgery to approximately $30-50 \mathrm{mg} / \mathrm{m}^{2} /$ day [16]. These small increases during uncomplicated minor surgery return to baseline in 24 hours. Procedures producing greater surgical stress (e.g., a spinal surgery) have been shown to increase cortisol responses to $75-150 \mathrm{mg} /$ day or $10 \mathrm{x}$ normal physiologic secretion, and can reach between 200 and $500 \mathrm{mg} /$ day in emergent surgery or severe trauma $[17,18]$. In most instances, basal cortisol secretion returns to base- 


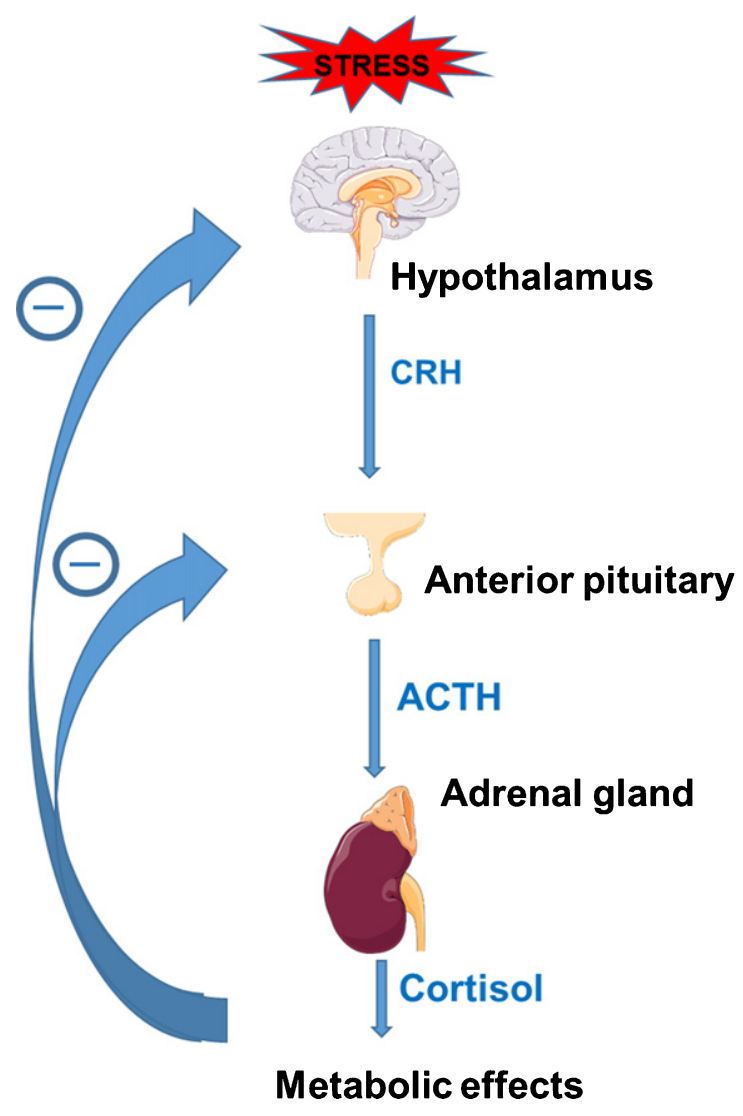

Fig. 1. Regulation through cortisol-mediated negative feedback of the hypothalamic-pituitary-adrenal (HPA) axis; $\mathrm{CRH}=$ corticotropin-releasing hormone, $\mathrm{ACTH}=$ adrenocorticotropin hormone. Figure designed with images from Servier Medical Art (https://smart.servier.com) under a Creative Commons Attribution 3.0 Unported License.

line about 5 days post operatively from these more complex surgeries [18].

The recommended daily dosage of $G C$ suppresses the HPA axis in DMD

Different types of synthetic GC preparations exert different pharmacologic effects based on their glucocorticoid, mineralocorticoid and anti-inflammatory activity. To determine the likelihood of adrenal suppression, one needs to consider the GC potency. Potency of different types of GC is measured using hydrocortisone as a reference, with potency value of 1 . Daily prednisone and deflazacort are prescribed for patients with DMD at $0.75 \mathrm{mg} / \mathrm{kg} /$ day and $0.9 \mathrm{mg} / \mathrm{kg} /$ day, respectively [19]. Prednisone has a relative potency of 4 when compared to hydrocortisone [20]. Therefore, if a boy with DMD who weighs $30 \mathrm{~kg}$ (body surface area of $1 \mathrm{~m}^{2}$ ) takes $22 \mathrm{mg}$ pred- nisone a day, the equivalent dose of hydrocortisone is $88 \mathrm{mg}(22 \times 4)$. This is approximately 8 times the physiologic dose of hydrocortisone $\left(10 \mathrm{mg} / \mathrm{m}^{2} /\right.$ day $)$. Prednisone $1 \mathrm{mg}$ is equivalent in anti-inflammatory effect to $1.2 \mathrm{mg}$ deflazacort [21]. Thus, for patients with DMD, the long term use of either daily prednisone or deflazacort at these recommended dosages that clearly exceed the physiologic dose of hydrocortisone would likely suppress the HPA axis, resulting in secondary AI [22].

\section{Adrenal insufficiency after long-term glucocorticoid therapy}

The Pediatric Endocrine Society recommends that adrenal suppression be considered in all children who have received supraphysiologic doses of oral daily $\mathrm{GC}$ ( $>12 \mathrm{mg} / \mathrm{m}^{2} /$ day hydrocortisone or equivalent) for two weeks or longer [11]. Long duration pharmacologic administration of synthetic GC, through negative feedback on the hypothalamus/pituitary (Fig. 1), leads to suppression of the HPA axis, with reduced production of $\mathrm{CRH}$ and $\mathrm{ACTH}$, resulting in secondary AI [22]. Suppression of the HPA axis causes decreased stimulation of the adrenal cortex and progressive partial or total atrophy of the adrenal glands, with decreased or absent adrenal reserve [22]. There is a great deal of individual variability in susceptibility to suppression of the HPA axis after withdrawal of chronic daily GC therapy [23, 24]. As a result, it is not possible to predict with confidence which patients will develop AI. Clinical variables including age, type and dosing regimen of GC, duration of treatment, cumulative GC dose, or time since GC withdrawal and basal cortisol levels are not predictive for $\mathrm{AI}$ assessed by peak cortisol on low-dose ACTH stimulation test [23-25]. In a recent study in Canada, adrenal crisis occurred in $13 \%$ of children with symptomatic adrenal suppression [26]. AI and/or adrenal crisis may result in significant morbidity and mortality, prolonged hospitalization and intensive care unit admission [27].

\section{Risk of adrenal crisis and adrenal insufficiency in $D M D$}

Patients treated with chronic GC who display cushingoid features should be assumed to have steroid-induced adrenal suppression [28, 29]. These patients show no signs of AI while on daily GC during the unstressed state, but may decompensate and develop acute AI when encountering physiological 
stress such as infection, surgery, or trauma, due to inability to produce sufficient additional cortisol to meet the stress demand. Common major surgery that would require GC stress dosing in DMD includes spinal surgery for scoliosis, tendon surgery for musculotendinous contractures, or open reduction for fractures of long bones. Surgery for scoliosis has become less frequent after the use of GC therapy in DMD [30]; recent study indicates that $20 \%$ of GCtreated group and $92 \%$ in the non GC-treated group developed scoliosis and underwent spinal surgery [31]. Prolonged treatment with GC results in osteoporosis, which increases the risk of low-trauma long bone fractures in patient with DMD. Recent studies indicate that $43 \%$ of patients living with DMD experience one or more fractures [32]. Both the experience of the fracture, as well as the possible need for surgical management, result in additional physiologic stress.

Patients with DMD may develop AI in these clinical circumstances:

1. AI can occur after abrupt withdrawal of GC after long-term therapy, whether voluntary cessation of therapy by patients, or if GC is inadvertently not given (i.e. hospitalization without continuation of GC), or due to illness that inhibits absorption of GC (e.g. gastroenteritis). Patients and caregivers must be educated about this risk and avoid discontinuing GC therapy without the physician guidance. Gradual tapering of GC doses allows the HPA axis to recover and also prevents steroid withdrawal or deprivation syndrome [33].

2. AI can also occur in patients encountering severe physiological stress events while on a GC dose that is inadequate to provide GC coverage for stress. For example, a patient weighing $30 \mathrm{~kg}$ (with body surface of $1.0 \mathrm{~m}^{2}$ ) taking $15 \mathrm{mg}$ of prednisone daily is on a dose that is above physiologic requirements and, therefore, would suppress the HPA axis. This patient would not experience AI symptoms as long as he takes prednisone daily. When he experiences mild to moderate stress, the prednisone dose, which is equivalent to $60 \mathrm{mg}$ hydrocortisone, is sufficient to provide moderate stress coverage. In the event of significant trauma or major surgery, this daily prednisone dose (or $60 \mathrm{mg}$ hydrocortisone) is insufficient to cover major stress, which would require $100 \mathrm{mg}$ hydrocortisone. Therefore, the patient would need additional GC to provide major stress coverage.

\section{Signs and symptoms of AI and adrenal crisis}

In patients with adrenal suppression, severe physiological stress can precipitate adrenal crisis, a life-threatening condition with ultimate signs of postural hypotension, dehydration, shock or coma. Clinicians must have high index of suspicion of AI in these patients, since early signs and symptoms of AI such as fatigue, nausea, vomiting, dizziness, or postural hypotension are non-specific and can be mistaken for symptoms of concurrent illness (such as viral gastroenteritis). Electrolyte abnormalities including hyponatremia and hypoglycemia can develop. Unlike primary AI (where hyperkalemia is common due to deficient aldosterone secretion), hyperkalemia is not observed in secondary AI [11].

\section{MANAGEMENT}

\section{Acute management of AI and adrenal crisis}

When encountering DMD patients with acute AI, immediate treatment with stress dose parenteral hydrocortisone, $100-150 \mathrm{mg}$ or $100 \mathrm{mg} / \mathrm{m}^{2}$ intravenously or intramuscularly, and saline bolus $(10 \mathrm{ml} / \mathrm{kg})$ is indicated. This is followed by intravenous hydrocortisone at stress dose (Fig. 2) given as continuous infusion over 24 hours or intravenous boluses divided into 4 doses (every 6 hours), along with intravenous isotonic saline with dextrose infusion to restore intravascular volume, and normalize serum sodium and glucose concentrations. Recognition and treatment of stress-precipitating conditions such as infection or trauma is also undertaken simultaneously. The stress hydrocortisone dose can be tapered over 2-3 days, if clinically appropriate, back to the home GC regimen for patients who are on chronic daily GC therapy. For patients who have abruptly discontinued long-term GC therapy, the stress hydrocortisone dose should be tapered to a physiologic hydrocortisone replacement therapy of $10-12.5 \mathrm{mg} / \mathrm{m}^{2} / \mathrm{day}$, and continued for a period of at least 1-2 months until further assessment of HPA axis is performed to ensure recovery of adrenal function. Physiologic replacement therapy, plus "stress doses" during physiological stress may need to be continued for a long period, as recovery of HPA suppression may take as long as 12-20 months [34]. 


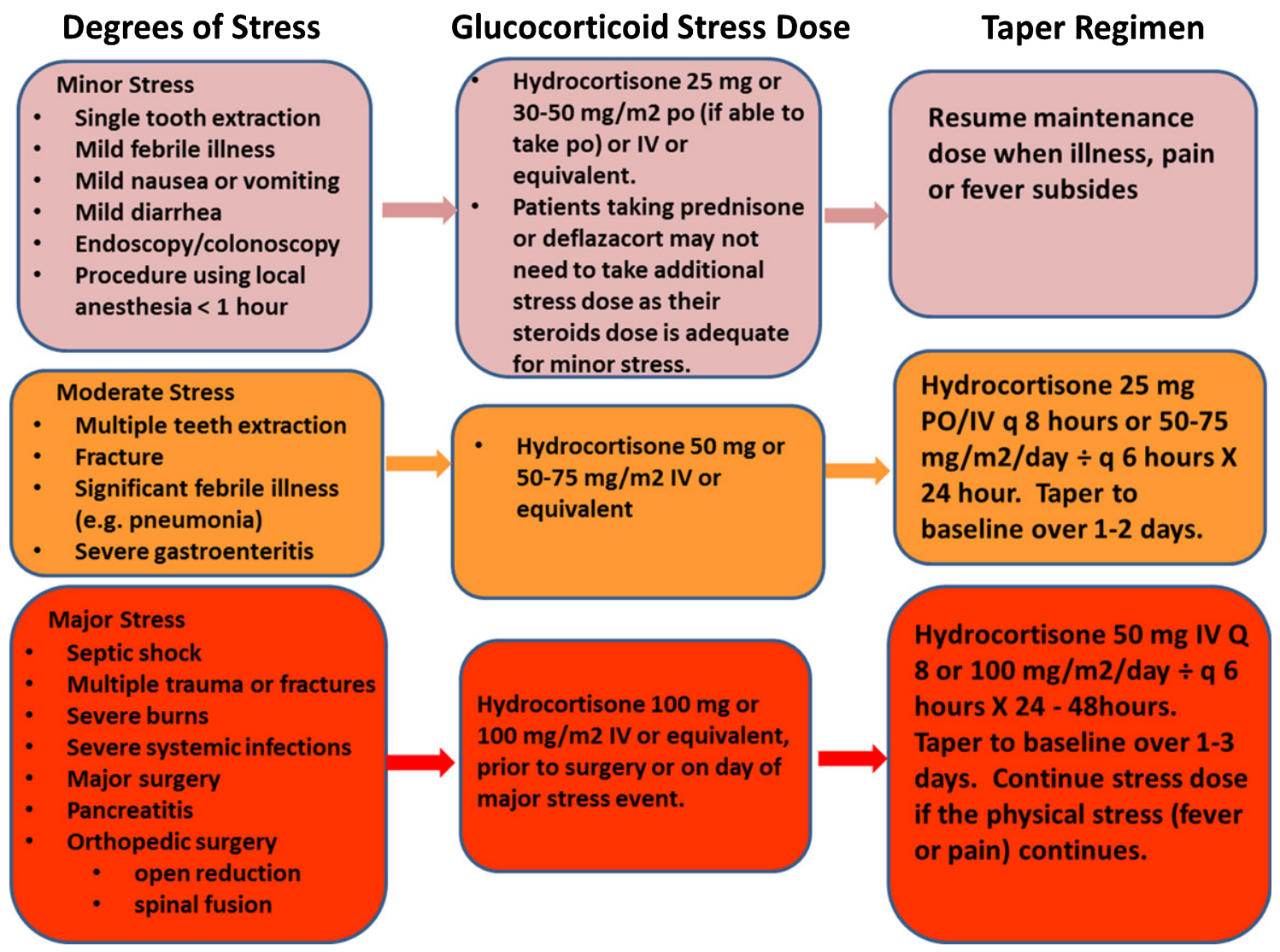

Fig. 2. Recommendations for steroids stress dose coverage for different degrees of stress [45].

Stress replacement during stressful events (illness, injury or surgery)

Cortisol secretion increases proportionate to the degree of stress [17]. A daily therapeutic dose of prednisone or deflazacort in boys with DMD generally provides sufficient coverage for mild to moderate stress, provided that the patient is able to take oral GC. However, this therapeutic dose is usually not sufficient for major stress (surgery, septic shock, multiple fractures), and the patients would require supplemental GC stress dosing (Fig. 2). The stress doses of GC can be rapidly reduced back to the therapeutic dose once clinically improved. If an illness causes patients to be unable to take oral medications, hydrocortisone must be administered parenterally at $25 \mathrm{mg} / \mathrm{m}^{2} /$ dose q 6 hours.

Some clinicians use intermittent GC regimens including twice weekly, or first 10 days of the month, or 10 days on and 10 days off. Twice weekly GC have not been associated with either growth arrest or cushingoid features [35]. While boys and men on these dosing regimens may be at lower risk of adrenal suppression or AI, this has not been formally studied. Therefore, the recommendations contained here should be considered.

\section{Recommendation for withdrawal of long-term glucocorticoid therapy}

Although long-term GC therapy is recommended in patients with DMD even after loss of ambulation to preserve cardiac and pulmonary function [7, 36, 37], some patients and/or medical providers still opt for discontinuation of therapy. There are no evidence-based guidelines on how to best taper GC after long-term daily therapy. A systematic review on GC withdrawal in chronic medical disorders found insufficient evidence of the efficacy and safety of the different withdrawal regimens [38]. Thus, recommendations for withdrawal of long-term GC therapy are based on expert opinion. There is a substantial individual variation in recovery rate of adrenal suppression. Clinicians need to monitor the patients 
Table 1

Prednisone or deflazacort tapering regimen

\section{Patients on daily prednisone or deflazacort:}

Step 1: Taper from pharmacologic to physiologic doses

1.1) Start on a Monday, reduce corticosteroids by $20-25 \%$ for 2 weeks (or longer).

1.2) Taper by $20-25 \%$ again every 2 weeks (or longer), until dose is near physiologic dose $\left(3 \mathrm{mg} / \mathrm{m}^{2} /\right.$ day or $0.1 \mathrm{mg} / \mathrm{kg} / \mathrm{day}$ of prednisone or $3.6 \mathrm{mg} / \mathrm{m}^{2} /$ day $0.12 \mathrm{mg} / \mathrm{kg} /$ day of deflazacort).

1.3) When near physiologic dose, switch prednisone or deflazacort to hydrocortisone at $12 \mathrm{mg} / \mathrm{m}^{2} /$ day, given twice a day, with higher dose (about $2 / 3$ of total daily dose) in the morning. Provide extra supply of hydrocortisone to be used for stress doses if needed in times of stress events.

1.4) Educate patients and caregivers on adrenal insufficiency and corticosteroid stress dosing.

1.5) Endocrinology outpatient consultation is highly recommended for patient education and evaluation of recovery of HPA axis.

Step 2: Taper from physiologic dose to complete withdrawal of corticosteroids

2.1) Continue to taper off hydrocortisone by $20-25 \%$ each week (or every 2 weeks).

2.2) If patients have symptoms of adrenal insufficiency during the taper, the previous steroid dose prior to the taper should be maintained for another week or longer.

2.3) Omit the evening dose when the dose is low. Give morning single dose for 1-2 weeks, then every other day for 2 weeks (or longer).

2.4) Discontinue the hydrocortisone and WATCH VERY CAREFULLY FOR SIGNS OF ADRENAL INSUFFICIENCY/CRISIS (Table 1).

2.5) If encountering a serious illness or injury during the taper, the patient should receive a "stress dose" of hydrocortisone (Figure 1).

2.6) Encourage parents to continue to report any serious physiological stress events (such as surgery) until 1 year post-taper to determine whether stress steroid dosing is necessary.

2.7) Educate patients and caregivers that they need to go to the emergency room if patients have signs or symptoms of adrenal crisis. Serum electrolytes, glucose and cortisol levels should be obtained.

closely and educate both patients and caregivers about the signs and symptoms of AI and slow the rate of tapering if needed. Table 1 outlines one recommendation for tapering chronic daily GC, with 2 important steps: first is to taper from pharmacologic dose to physiologic dose; second step is to taper from physiologic dose to discontinuation. When the GC dose is near or below physiologic, it is critical to monitor for signs or symptoms of adrenal insufficiency, especially when patients encounter stress events. It is therefore recommended that during the process of tapering in step 1, outpatient endocrinology consultation be obtained to establish care, provide teaching on $\mathrm{AI}$ as well as stress dosing, and to plan for adrenal function testing after the discontinuation of GC. Clinicians should have a low threshold to test and treat for AI, especially when patients develop symptoms suggestive of AI during the tapering and after cessation of GC. Written information on AI and adrenal suppression with steroid emergency card (Fig. 3) should also be provided to patients and caregivers.

\section{Assessing recovery of the \\ hypothalamic-pituitary-adrenal axis}

There have been no studies assessing adrenal function and timing of recovery in patients with DMD after long-term GC therapy. In a study of children treated with long-term GC therapy for rheumatic disease, AI based on inadequate response to low-dose ACTH stimulation test was found in $32 \%$ and $11 \%$ at

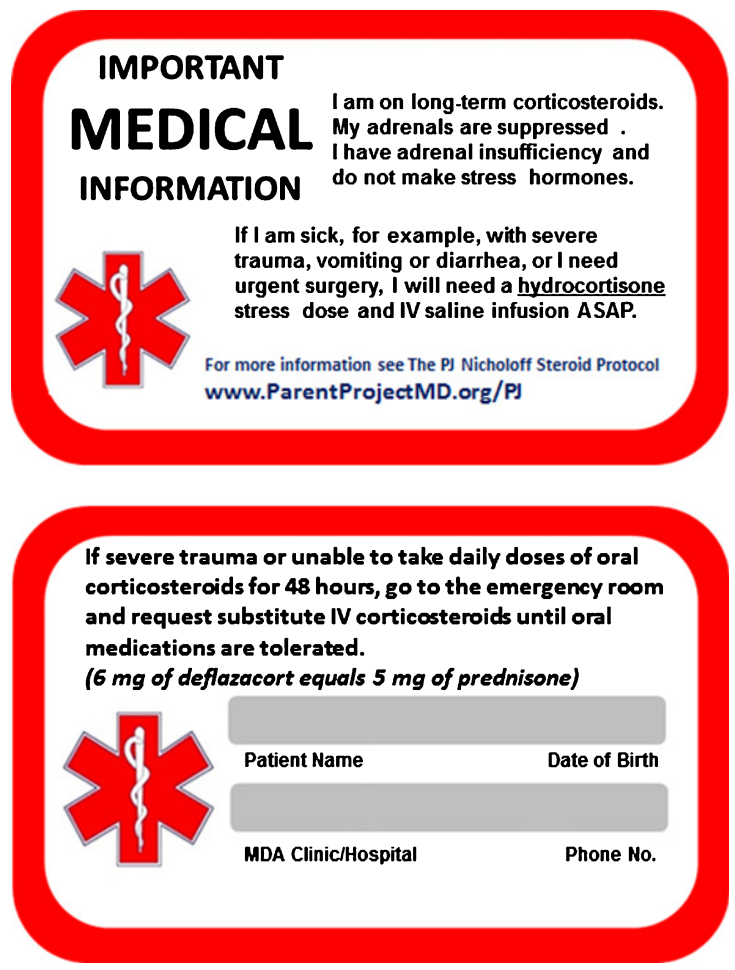

Fig. 3. Steroid emergency card, front and back. A complete DMD emergency card that includes all aspect of care for patients with DMD is available at the Parent Project Muscular Dystrophy website [49].

1 month and 20 months, respectively, after discontinuation of GC [34]. A study by Graber et al evaluating 
Table 2

Assessment of hypothalamic-pituitary axis after corticosteroid withdrawal. After reaching half the physiologic dose $\left(5-6 \mathrm{mg} / \mathrm{m}^{2} /\right.$ day of hydrocortisone) or less, obtain morning cortisol monthly to determine if hydrocortisone can be discontinued

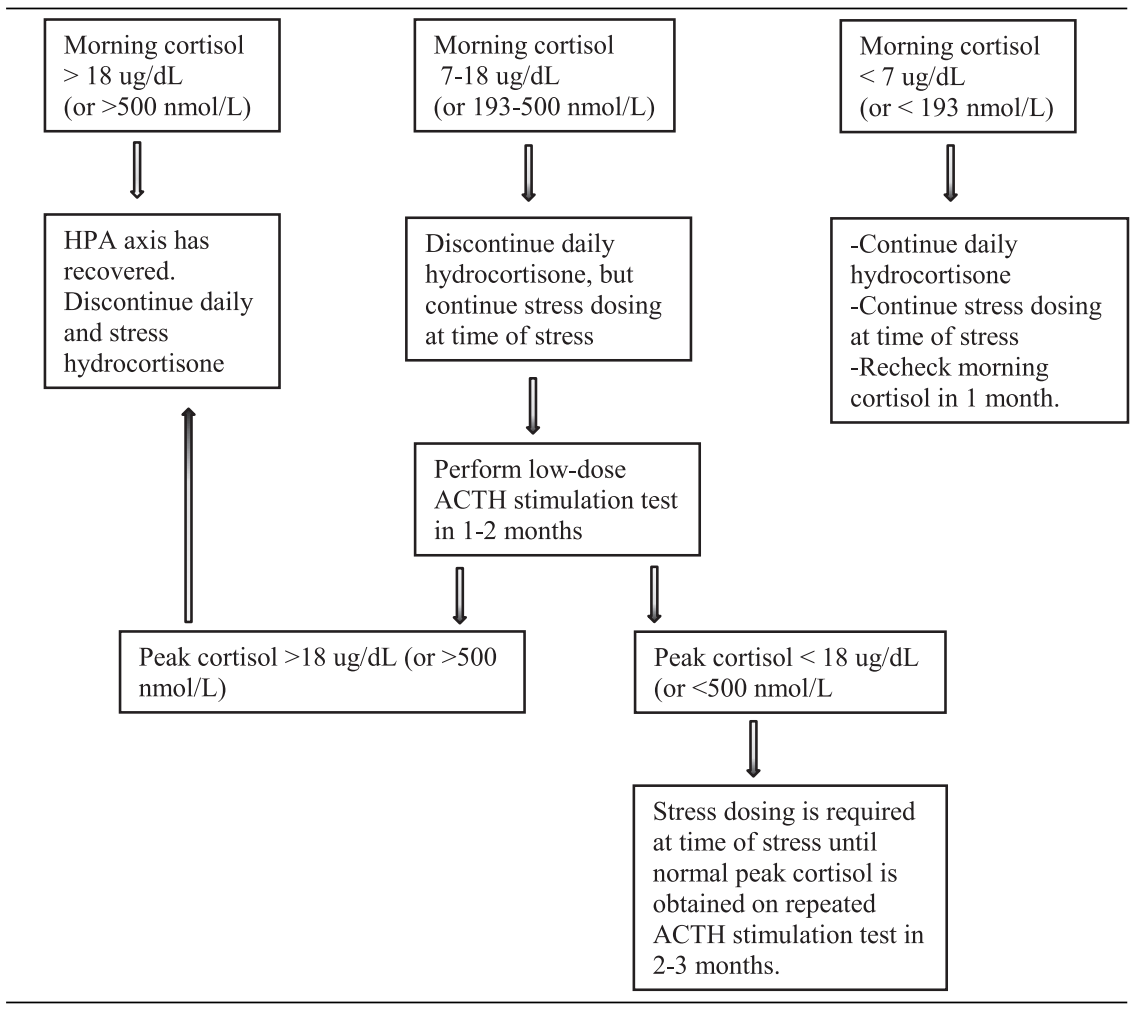

recovery of the HPA axis after withdrawal of chronic GC therapy demonstrated that ACTH was the first to normalize (after $2-5$ months), followed by morning serum cortisol (after 6-9 months), and finally cortisol after adrenal stimulation testing with ACTH (after 9 months), which is thought to best reflect response to physiologic stress [39]. Based on this knowledge, it is recommended that assessment of morning cortisol be obtained when the tapering process is reaching half the physiologic dose or less to determine if hydrocortisone can be discontinued [40], as guided by morning cortisol values outlined in Table 2 . The HPA axis is intact and daily hydrocortisone is discontinued when morning cortisol is $>18 \mu \mathrm{g} / \mathrm{dL}$ (or $>500 \mathrm{nmol} / \mathrm{L}$ ). When morning cortisol is between $7-18 \mu \mathrm{g} / \mathrm{dL}$, adrenal function is likely adequate for non-stressed state, but may be inadequate for stress. Thus, daily hydrocortisone is discontinued but stress dosing at time of stress is continued until a low-dose ACTH stimulation test (protocol shown in Table 3) is obtained to document recovery of the HPA axis [41]. Alternatively, clinicians may skip morning cor- tisol testing and perform ACTH stimulation test 2-3 months after discontinuation of hydrocortisone. In the absence of evidence-based guidelines, clinicians may choose to gradually taper off GC therapy without HPA testing as described above, with great vigilance for signs and symptoms of AI. It is also a safe approach to consider patients as having adrenal suppression for up to 1 year after discontinuation of GC therapy and to provide prophylactic therapy with hydrocortisone when there is any concern for AI, especially at time of stress.

\section{Special considerations for non-daily glucocorticoid therapy in DMD}

The dosing and type of corticosteroid use varies widely within the United States and around the world. In the United States, besides the daily prednisone and daily deflazacort regimens, twice weekly only prednisone at $5 \mathrm{mg} / \mathrm{kg}$ twice weekly ( $10 \mathrm{mg} / \mathrm{kg} /$ week) has also been used and has been shown to be equally effective as daily prednisone in preserving muscle 
Table 3

Protocol for low-dose ACTH stimulation test to assess HPA axis

\footnotetext{
1. Fasting is not necessary.

2. On arrival obtain height and weight, and vital signs.

3. If patient desires, lidocaine $4 \%$ cream (2.5 gram) can be applied to site 20-30 minutes prior to intravenous (IV) insertion.

4. Place saline lock $-0.9 \%$ NS $10 \mathrm{~mL}$ flush for blood draws and after IV medications.

5. Obtain baseline blood sample for cortisol at $(t=0)$.

6. Prepare cortrosyn dilution: Dilute $250 \mathrm{mcg}$ into $10 \mathrm{ml}$ normal saline, mix well. Each $0.04 \mathrm{ml}$ (4 units on insulin syringe) contains 1 microgram cortrosyn.

7. Give cortrosyn $1 \mathrm{mcg} I \mathrm{~V}$ at $t=0$ minute. Inject as close as possible to the IV catheter and immediately flush with normal saline to avoid adherence of cortrosyn to IV tubing.

8. Draw cortisol at $t=20,40$ minutes.
}

strength over 12-month period [35]. Despite the fact that linear growth is maintained and cushingoid features are not observed, even after years [42], it is prudent to consider, evaluate, and treat for AI during stress. While formal adrenal testing has not been performed routinely, ACTH stimulation tests performed in children prior to surgery have shown normal results (AMC personal communication).

Efficacy of daily prednisone at $0.75 \mathrm{mg} / \mathrm{kg} /$ day 10 days on/10 days off commonly used in Europe has also been reported [43] and again, linear growth is preserved and cushingoid features are less common. Again, there have been no published studies examining the extent of adrenal suppression during the off days in the patients on these regimens. It is prudent to assume that the patients may have adrenal suppression. We recommend that if patients are unable to take GC by mouth due to nausea, vomiting, or need to be fasted for a procedure, the patients should be given stress doses intravenously as indicated in Fig. 2. When these patients encounter stress events during the off days, stress dose hydrocortisone may be given if clinically indicated. If the patients experience signs and symptoms of AI, or encounter major stress, serum cortisol level may be drawn prior to stress dosing, if possible, to document AI (cortisol level $<18 \mathrm{ug} / \mathrm{dL}$ or $<500 \mathrm{nmol} / \mathrm{L}$ during stress event would suggest AI). Future studies to evaluate adrenal function in patients on intermittent $\mathrm{GC}$ regimens are needed.

\section{Strategies to increase awareness of adrenal insufficiency and translate guidelines into practice improvements}

Morbidity and mortality associated with AI can be prevented by increased recognition and awareness of adrenal suppression and risk of $\mathrm{AI}$ and adrenal crisis among patients, caregivers and health care providers.
Recently a death related to AI in a patient with DMD has catalyzed the development of a protocol named "PJ Nicholoff Steroid Protocol" in memory of the patient [13]. This protocol, developed through an effort by Parent Project Muscular Dystrophy and St. Vincent's Hospital of Indianapolis, is available on the Parent Project Muscular Dystrophy (ParentprojectMD.org) and Muscular Dystrophy (MDA.org) websites [46, 47]. How these recommendations or guidelines translate into practice and improve outcome has yet to be studied. A recent prospective study showed that the incidence of adrenal crisis remained high due to failure to properly administer GC stress doses among patients with chronic AI, despite having prior education [44]. This study highlights the need to identify effective strategies to improve education among patients and caregivers and to improve quality of care delivery related to AI by health care providers. We have designed a quality-improvement diagram (Fig. 4) that include the aim statement, key drivers and the interventions to help increase awareness and education on AI and adrenal suppression. We encourage physicians caring for DMD patients to adopt this quality-improvement project and make both the emergency card (Fig. 3) and mobile app [48] available to patients seen in their practice, as well as encouraging patients to wear medical ID alerting first responders to their diagnosis, allergies and medications (i.e., "daily corticosteroids"). One important strategy that needs to be emphasized is to add "Steroid-induced adrenal suppression" to the "Problem List" in the patient's medical record, to alert physicians caring for patients in the urgent care facility or emergency department for the risk of AI. It is also important for the muscular dystrophy care team to provide families with a method to link them with the neuromuscular team in case of an emergency, and to re-assess patients' and caregivers' understanding and knowledge on AI risks at each clinic visit. 


\section{Interventions}

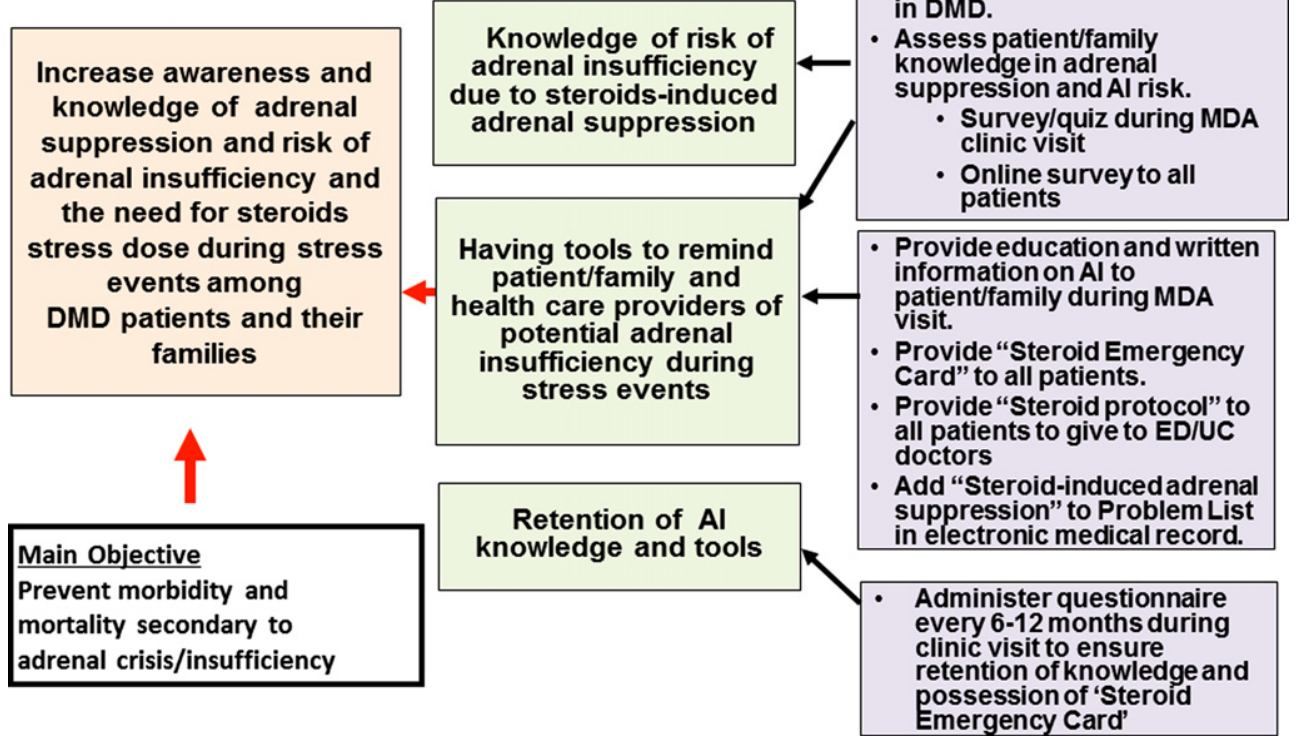

Fig. 4. Key drivers for our quality-improvement project. Shown is the learning structure including the aim statement, key drivers, and the intervention strategies to be implemented to increase awareness of adrenal suppression and risk of adrenal insufficiency or adrenal crisis. The key drivers are the elements believed to be crucial for achieving the aim.

\section{CONCLUSIONS}

Chronic GC therapy causes adrenal suppression in patients with DMD, therefore, clinicians must have high index of suspicion for AI and GC stress dosing should be given to patients at the time of significant physiologic stress events. Education regarding AI risks must be provided to caregivers and patients with DMD on long-term GC therapy to prevent morbidity and mortality associated with AI or adrenal crisis.

\section{FUNDING SOURCE}

This work was completed with no specific funding.

\section{DISCLOSURE STATEMENT}

The authors declare that there is no conflict of interests regarding the publication of this article.

\section{ACKNOWLEDGMENTS}

We thank Dr Douglas Biggar for helpful comments.

\section{REFERENCES}

[1] Brooke M, Fenichel G, Griggs R, Mendell J, Moxley R, Florence J, et al. Duchenne muscular dystrophy Patterns of clinical progression and effects of supportive therapy. Neurology. 1989;39(4):475.

[2] Mah JK, Korngut L, Dykeman J, Day L, Pringsheim T, Jette N. A systematic review and meta-analysis on the epidemiology of Duchenne and Becker muscular dystrophy. Neuromuscular Disorders. 2014;24(6):482-91.

[3] Emery AE. Population frequencies of inherited neuromuscular diseases-a world survey. Neuromuscular disorders : NMD. 1991;1(1):19-29.

[4] Romitti PA, Zhu Y, Puzhankara S, James KA, Nabukera SK, Zamba GK, et al. Prevalence of Duchenne and Becker muscular dystrophies in the United States. Pediatrics. 2015;135(3):513-21.

[5] Mendell JR, Shilling C, Leslie ND, Flanigan KM, al-Dahhak $\mathrm{R}$, Gastier-Foster J, et al. Evidence-based path to newborn screening for Duchenne muscular dystrophy. Annals of Neurology. 2012;71(3):304-13.

[6] Moxley RT, Pandya S, Ciafaloni E, Fox DJ, Campbell $\mathrm{K}$. Change in natural history of Duchenne muscular dystrophy with long-term corticosteroid treatment: implications for management. Journal of Child Neurology. 2010.

[7] Connolly AM, Malkus EC, Mendell JR, Flanigan KM, Miller JP, Schierbecker JR, et al. Outcome reliability in nonAmbulatory Boys/Men with duchenne muscular dystrophy. Muscle \& Nerve. 2015;51(4):522-32.

[8] Matthews E, Brassington R, Kuntzer T, Jichi F, Manzur AY. Corticosteroids for the treatment of Duchenne muscular dystrophy. The Cochrane Library. 2016. 
[9] Drozdowicz LB, Bostwick JM, editors. Psychiatric Adverse Effects of Pediatric Corticosteroid Use. Mayo Clinic Proceedings; 2014: Elsevier.

[10] Gloss D, Moxley 3rd R, Ashwal S, Oskoui M. Practice guideline update summary: Corticosteroid treatment of Duchenne muscular dystrophy: Report of the Guideline Development Subcommittee of the American Academy of Neurology. Neurology. 2016;86(5):465-72.

[11] Shulman DI, Palmert MR, Kemp SF. Adrenal insufficiency: still a cause of morbidity and death in childhood. Pediatrics. 2007;119(2):e484-e94.

[12] Puar TH, Stikkelbroeck NM, Smans LC, Zelissen PM, Hermus AR. Adrenal crisis: still a deadly event in the 21st century. The American Journal of Medicine. 2016;129(3):339. e1-e9.

[13] Kinnett K, Noritz G. The PJ Nicholoff Steroid Protocol for Duchenne and Becker Muscular Dystrophy and Adrenal Suppression. PLoS Currents. 2017;9.

[14] Kerrigan JR, Veldhuis JD, Leyo SA, Iranmanesh A, Rogol AD. Estimation of daily cortisol production and clearance rates in normal pubertal males by deconvolution analysis. The Journal of Clinical Endocrinology \& Metabolism. 1993;76(6):1505-10.

[15] Esteban NV, Loughlin T, Yergey AL, Zawadzki JK, Booth JD, Winterer JC, et al. Daily cortisol production rate in man determined by stable isotope dilution/mass spectrometry. The Journal of Clinical Endocrinology \& Metabolism. 1991;72(1):39-45.

[16] Wood AJ, Lamberts SW, Bruining HA, de Jong FH. Corticosteroid therapy in severe illness. New England Journal of Medicine. 1997;337(18):1285-92.

[17] Lamberts SW, Bruining HA, de Jong FH. Corticosteroid therapy in severe illness. New England Journal of Medicine. 1997;337(18):1285-92.

[18] Yong SL, Coulthard P, Wrzosek A. Supplemental perioperative steroids for surgical patients with adrenal insufficiency. Cochrane Database Syst Rev. 2012;12.

[19] Gloss D, Moxley RT, 3rd, Ashwal S, Oskoui M. Practice guideline update summary: Corticosteroid treatment of Duchenne muscular dystrophy: Report of the Guideline Development Subcommittee of the American Academy of Neurology. Neurology. 2016;86(5):465-72.

[20] Meikle AW, Tyler FH. Potency and duration of action of glucocorticoids: effects of hydrocortisone, prednisone and dexamethasone on human pituitary-adrenal function. The American Journal of Medicine. 1977;63(2):200-7.

[21] Gonzalez-Perez O, Luquin S, Garcia-Estrada J, RamosRemus C. Deflazacort: a glucocorticoid with few metabolic adverse effects but important immunosuppressive activity. Advances In Therapy. 2007;24(5):1052-60.

[22] Krasner AS. Glucocorticoid-induced adrenal insufficiency. JAMA. 1999;282(7):671-6.

[23] Wildi-Runge S, Deladoëy J, Bélanger C, Deal CL, Van Vliet G, Alos N, et al. A Search for Variables Predicting Cortisol Response to Low-Dose Corticotropin Stimulation Following Supraphysiological Doses of Glucocorticoids. The Journal of Pediatrics. 2013;163(2):484-8.e1.

[24] Dinsen S, Baslund B, Klose M, Rasmussen AK, FriisHansen L, Hilsted L, et al. Why glucocorticoid withdrawal may sometimes be as dangerous as the treatment itself. European Journal of Internal Medicine. 2013;24(8):714-20.

[25] Felner EI, Thompson MT, Ratliff AF, White PC, Dickson BA. Time course of recovery of adrenal function in children treated for leukemia. The Journal of Pediatrics. 2000;137(1):21-4.
[26] Goldbloom EB, Mokashi A, Cummings EA, Abish S, Benseler SM, Huynh HQ, et al. Symptomatic adrenal suppression among children in Canada. Archives of Disease in Childhood. 2016:archdischild-2016-311223.

[27] Rix M, Birkebæk NH, Rosthøj S, Clausen N. Clinical impact of corticosteroid-induced adrenal suppression during treatment for acute lymphoblastic leukemia in children: a prospective observational study using the lowdose adrenocorticotropin test. The Journal of Pediatrics. 2005;147(5):645-50.

[28] Perry R, Findlay C, Donaldson M. Cushing's syndrome, growth impairment, and occult adrenal suppression associated with intranasal steroids. Archives of Disease in Childhood. 2002;87(1):45-8.

[29] Lansang MC, Hustak LK. Glucocorticoid-induced diabetes and adrenal suppression: how to detect and manage them. Cleveland Clinic Journal of Medicine. 2011;78(11):748-56.

[30] Raudenbush BL, Thirukumaran CP, Li Y, Sanders JO, Rubery PT, Mesfin A. Impact of a Comparative Study on the Management of Scoliosis in Duchenne Muscular Dystrophy: Are Corticosteroids Decreasing the Rate of Scoliosis Surgery in the United States? Spine. 2016;41(17):E1030E8.

[31] Lebel DE, Corston JA, McAdam LC, Biggar WD, Alman BA. Glucocorticoid treatment for the prevention of scoliosis in children with Duchenne muscular dystrophy: long-term follow-up. The Journal of Bone \& Joint Surgery. 2013;95(12):1057-61.

[32] Perera N, Sampaio H, Woodhead H, Farrar M. Fracture in Duchenne muscular dystrophy: natural history and vitamin D deficiency. Journal of Child Neurology. 2016;31(9):11817.

[33] Margolin L, Cope DK, Bakst-Sisser R, Greenspan J. The steroid withdrawal syndrome: a review of the implications, etiology, and treatments. Journal of Pain and Symptom Management. 2007;33(2):224-8.

[34] Huber BM, Bolt IB, Sauvain M-J, Flück CE. Adrenal insufficiency after glucocorticoid withdrawal in children with rheumatic diseases. ACTA Paediatrica. 2010;99(12):188993.

[35] Escolar D, Hache L, Clemens P, Cnaan A, McDonald C, Viswanathan V, et al. Randomized, blinded trial of weekend vs daily prednisone in Duchenne muscular dystrophy. Neurology. 2011;77(5):444-52.

[36] Connolly AM, Florence JM, Zaidman CM, Golumbek PT, Mendell JR, Flanigan KM, et al. Clinical trial readiness in non-ambulatory boys and men with duchenne muscular dystrophy: MDA-DMD network follow-up. Muscle \& Nerve. 2016;54(4):681-9.

[37] Finder J, Mayer OH, Sheehan D, Sawnani H, Abresch RT, Benditt J, et al. Pulmonary Endpoints in Duchenne Muscular Dystrophy. A Workshop Summary. American Journal of Respiratory and Critical Care Medicine. 2017;196(4):5129.

[38] Richter B, Neises G, Clar C. Glucocorticoid withdrawal schemes in chronic medical disorders: A systematic review. Endocrinology and Metabolism Clinics of North America. 2002;31(3):751-78.

[39] Graber AL, Ney RL, Nicholson WE, Island DP, Liddle GW. Natural history of pituitary-adrenal recovery following long-term suppression with corticosteroids. The Journal of Clinical Endocrinology \& Metabolism. 1965;25(1):11-6.

[40] Hägg E, Asplund K, Lithner F. Value of basal plasma cortisol assays in the assessment of pituitary-adrenal insufficiency. Clinical Endocrinology. 1987;26(2):221-6. 
[41] Oelkers W. The role of high-and low-dose corticotropin tests in the diagnosis of secondary adrenal insufficiency. European Journal of Endocrinology. 1998;139(6):567-70.

[42] Connolly AM, Schierbecker J, Renna R, Florence J. High dose weekly oral prednisone improves strength in boys with Duchenne muscular dystrophy. Neuromuscular Disorders. 2002;12(10):917-25.

[43] Straathof CS, Overweg-Plandsoen WT, van den Burg GJ, van der Kooi AJ, Verschuuren JJ, de Groot IJ. Prednisone 10 days on/10 days off in patients with Duchenne muscular dystrophy. Journal of Neurology. 2009;256(5):768-73.

[44] Hahner S, Spinnler C, Fassnacht M, Burger-Stritt S, Lang $\mathrm{K}$, Milovanovic D, et al. High incidence of adrenal crisis in educated patients with chronic adrenal insufficiency: a prospective study. The Journal of Clinical Endocrinology \& Metabolism. 2015;100(2):407-16.

[45] Coursin DB, Wood KE. Corticosteroid supplementation for adrenal insufficiency. JAMA. 2002;287(2):236-40.

[46] www.ParentProjectMD.org/PJ

[47] https://www.mda.org/sites/default/files/PJ_Nicholoff_Stero id_Protocol.pdf

[48] http://www.parentprojectmd.org/site/PageServer?pagename $=$ Connect_mobile

[49] http://www.parentprojectmd.org/site/PageServer?pagename $=$ Care_resources_materials_emergencyinfo 